\title{
EXISTENCE AND NONEXISTENCE OF GLOBAL SOLUTIONS TO FAST DIFFUSIONS WITH SOURCE
}

\author{
Kiyoshi Mochizuki and Kentaro Mukai
}

\begin{abstract}
We consider positive solutions to the Cauchy problem for quasilinear parabolic equations $\partial_{t} u=\Delta u^{m}+u^{p}$ with $\max \{0,1-2 / N\}<m<1<p$, where $N$ is the space dimension. Putting $p_{m}^{*}=m+2 / N$, we shall show that if $p \leq p_{m}^{*}$, then all nontrivial solutions blow up in finite time, and if $p>p_{m}^{*}$, then there are nontrivial global solutions.
\end{abstract}

\section{Introduction}

We consider the Cauchy problem

$$
\begin{gathered}
\partial_{t} u=\Delta u^{m}+u^{p}, \quad(x, t) \in \mathbf{R}^{N} \times(0, T), \\
u(x, 0)=u_{0}(x), \quad x \in \mathbf{R}^{N},
\end{gathered}
$$

where $0<m<1<p$ and $u_{0}(x) \geq 0$. Equation (1) with $0<m<1$ arises in the plasma physics (see Berryman [1]). Since the thermal conductivity $m u^{m-1} \uparrow \infty$ when $u \downarrow 0$, mathematically (1) represents a fast diffusion with source (Peletier [11]).

In the case of slow diffusion $m \geq 1$, the following result is known to hold. Let

$$
p_{m}^{*}=m+\frac{2}{N}
$$

If $1<p \leq p_{m}^{*}$, then all nontrivial nonnegative solutions of (1)-(2) blow up in finite time, whereas if $p>p_{m}^{*}$, then global solutions of (1)-(2) exist when the initial data are sufficiently small (see Fujita [5], Hayakawa [8], and Weissler [14] for $m=1$, and Galaktionov et al. [7], Galaktionov [6], Kawanago [9], and Mochizuki-Suzuki [10] for $m>1$ ). Thus, the number $p_{m}^{*}$ is the cutoff between the blow-up case and the global existence case, and it is called the critical exponent.

Similar blow-up and global existence results are expected to hold also for the case of fast diffusion $m<1$.

In this paper, we restrict ourselves to the case

$$
\max \left\{0,1-\frac{2}{N}\right\}<m<1,
$$

and we assume that $u_{0}(x)$ is continuous in $x \in \mathbf{R}^{N}$ and

$$
u_{0}(x) \in L^{1}\left(\mathbf{R}^{N}\right) \cap L^{\infty}\left(\mathbf{R}^{N}\right) .
$$

Then a unique weak solution $u(x, t) \in C\left([0, T) ; L^{1}\left(\mathbf{R}^{N}\right)\right) \cap L_{\text {loc }}^{\infty}\left([0, T) ; L^{\infty}\left(\mathbf{R}^{N}\right)\right)$ of (1)-(2) exists at least for sufficiently small $T>0$ (see, e.g., Brézis-Crandall [2]), and

Received June 27, 1994, revised September 2, 1994. 
it becomes continuous in $(x, t) \in \mathbf{R}^{N} \times[0, T)$. When $T=\infty$, we say $u$ is global; otherwise we say $u$ blows up in finite time. In the latter case, it follows that

$$
\|u(\cdot, t)\|_{L^{\infty}} \rightarrow \infty \quad \text { as } \quad t \uparrow T .
$$

We shall prove the following theorems.

Theorem 1.1. If $1<p \leq p_{m}^{*}$, then all positive solutions of the Cauchy problem (1)-(2) blow up in finite time.

Theorem 1.2. If $p>p_{m}^{*}$, then for sufficiently small $u_{0}(x) \in L^{\infty}\left(\mathbf{R}^{N}\right)$ such that

$$
u_{0}(x)=O\left(|x|^{-2 /(1-m)}\right) \text { as } \quad|x| \rightarrow \infty,
$$

the Cauchy problem (1)-(2) has a global solution with

$$
\sup _{x \in \mathbf{R}^{N}} u(x, t) \leq C t^{-1 /(p-1)},
$$

where $C$ is some positive constant.

The proofs of the above theorems depend on a comparison principle for parabolic equations (Proposition 2.1). To show Theorem 1.2, we shall construct a supersolution of (1)-(2) which behaves like $O\left(t^{-1 /(p-1)}\right)$ as $t \rightarrow \infty$ (cf. [7]). To show Theorem 1.1, we shall modify the argument of [10] with the aid of a result of Friedman-Kamin [4]. The subcritical case $1<p<p_{m}^{*}$ being easy, we explain our argument in the critical case $p=p_{m}^{*}$.

Let $u$ be a nontrivial global solution to (1)-(2). Then our blow-up condition (Proposition 2.3) implies

$$
\int_{0}^{t} \int_{\mathbf{R}^{N}} u(x, \tau)^{p} d x d \tau \leq \pi^{N / 2}(2 N)^{1 /(p-m)} \text { for } \quad t>0 .
$$

If $m>1$, then, as is proved in [10], one can find a Barenblatt solution $E_{m}(x, t ; L)$ of the porous media equation to satisfy $E_{m}\left(x, t_{1} ; L\right) \leq u_{0}(x)$ for some $t_{1}>0$. This leads to a contradiction since we have

$$
\int_{t_{1}}^{t} \int_{\mathbf{R}^{N}} E_{m}(x, \tau ; L)^{p} d x d \tau \rightarrow \infty \quad \text { as } \quad t \rightarrow \infty .
$$

In our fast diffusion problem, however, the support of every Barenblatt solution spreads out to the whole $\mathbf{R}^{N}$ whenever $t>0$, and it becomes difficult to find such a convenient subsolution to (1)-(2). To remove this difficulty, we turn our attention to the self-similarity of the Barenblatt solutions,

$$
E_{m}(x, t ; L)=k^{N} E_{m}\left(k x, k^{N / \ell} t ; L\right), \quad k>0,
$$

where $\ell=\left(p_{m}^{*}-1\right)^{-1}$. We put $u_{k}(x, t)=k^{N} u\left(k x, k^{N / \ell} t\right)$. Then it also gives a global solution to (1)-(2) with $u_{0}(x)$ replaced by $k^{N} u_{0}(k x)$. Compare this $u_{k}(x, t)$ and $E_{m}(x, t ; L)$ with $L=\int_{\mathbf{R}^{N}} u_{0}(x) d x$, when $k \rightarrow \infty$. Then applying the asymptotic behavior for porous media equations (cf., [4, Remark 2]), we reach a similar contradiction.

Note here that our results can be extended to the exterior Dirichlet boundary-value problem if $N \geq 3$. In his recent work [13], R. Suzuki has obtained a critical blow-up 
to the exterior problem of slow diffusion. His argument is applicable also to our fast diffusion problem without any essential modification.

The rest of the paper is organized as follows. In $\S 2$, we define a weak solution of (1) and prepare several preliminary propositions. Theorems 1.1 and 1.2 are proved in $\S 3$ and $\S 4$, respectively. Finally in $\S 5$, we remark on a critical blow-up for the exterior problem.

\section{Preliminaries}

We begin with the definition of a weak solution of (1).

Definition 2.1. By a solution of equation (1) we mean a function $u(x, t)$ in $\mathbf{R}^{N} \times[0, T)$ such that

(i) $u(x, t) \geq 0$ and $\in C\left(\left[0, T^{\prime}\right] ; L^{1}\left(\mathbf{R}^{N}\right)\right) \cap L^{\infty}\left(\mathbf{R}^{N} \times\left[0, T^{\prime}\right]\right)$ for any $0<T^{\prime}<T$.

(ii) For any bounded $G \subset \mathbf{R}^{N}, 0 \leq \tau<T$ and nonnegative $\varphi(x, t) \in C^{2}(\bar{G} \times[0, T))$ which vanishes on the boundary $\partial G$,

$$
\begin{aligned}
\int_{G} u(x, \tau) & \varphi(x, \tau) d x-\int_{G} u(x, 0) \varphi(x, 0) d x \\
= & \int_{0}^{\tau} \int_{G}\left\{u \varphi_{t}+u^{m} \Delta \varphi+u^{p} \varphi\right\} d x d t-\int_{0}^{\tau} \int_{\partial G} u^{m} \partial_{n} \varphi d S d t
\end{aligned}
$$

where $n$ denotes the outer unit normal to the boundary.

A supersolution [or subsolution] is similarly defined with equality (8) replaced by $\geq[$ or $\leq]$.

The following comparison principle is well-known for the quasilinear equation (1). The result will be freely used in the sequel.

Proposition 2.1. Let $u$ [or $v$ ] be a supersolution [or subsolution] of $(1)$. If $u(x, 0) \geq$ $v(x, 0)$, then we have $u \geq v$ in the whole $\mathbf{R}^{N} \times[0, T)$.

Let $\varphi(x, t) \in C^{2}\left(\mathbf{R}^{N} \times[0, T)\right)$ satisfy

$$
\int_{\mathbf{R}^{N}}\left\{\varphi+\left|\partial_{t} \varphi\right|+|\nabla \varphi|+|\Delta \varphi|\right\} d x<\infty
$$

Then, by a limit procedure, we have from (8),

$$
\begin{aligned}
& \int_{\mathbf{R}^{N}} u(x, \tau) \varphi(x, \tau) d x-\int_{\mathbf{R}^{N}} u(x, 0) \varphi(x, 0) d x \\
&=\int_{0}^{\tau} \int_{\mathbf{R}^{N}}\left\{u \varphi_{t}+u^{m} \Delta \varphi+u^{p} \varphi\right\} d x d t .
\end{aligned}
$$

We put $\varphi=e^{-\epsilon|x|^{2}}, \epsilon>0$, in this equation. Then it follows that

$$
\begin{array}{rl}
\int_{\mathbf{R}^{N}} u(x, \tau) e^{-\epsilon|x|^{2}} & d x-\int_{\mathbf{R}^{N}} u(x, 0) e^{-\epsilon|x|^{2}} d x \\
& =\int_{0}^{\tau} \int_{\mathbf{R}^{N}}\left\{\left(-2 N \epsilon+4 \epsilon^{2}|x|^{2}\right) u^{m}+u^{p}\right\} e^{-\epsilon|x|^{2}} d x d t .
\end{array}
$$

Our proof of Theorem 1.1 will be based on the following two propositions. 
Proposition 2.2. Let $u$ be a solution to (1)-(2). Then for any $\tau \in(0, T)$,

$$
\int_{\mathbf{R}^{N}} u(x, \tau) d x-\int_{\mathbf{R}^{N}} u_{0}(x) d x=\int_{0}^{\tau} \int_{\mathbf{R}^{N}} u(x, t)^{p} d x d t .
$$

Moreover, we have

$$
\int_{\mathbf{R}^{N}} u(x, t) d x \leq e^{a(\tau) t} \int_{\mathbf{R}^{N}} u_{0}(x) d x \quad \text { for } \quad t \in[0, \tau]
$$

where

$$
a(\tau)=\sup _{(x, t) \in \mathbf{R}^{N} \times(0, \tau)} u(x, t)^{p-1}
$$

Proof. Note that

$$
\begin{aligned}
& \left|\int_{\mathbf{R}^{N}}\left(-2 N \epsilon+4 \epsilon^{2}|x|^{2}\right) u^{m} e^{-\epsilon|x|^{2}} d x\right| \leq \epsilon^{1-(1-m) N / 2}\left(\int_{\mathbf{R}^{N}} u d x\right)^{m} \\
& \quad \times\left\{2 N\left(\int_{\mathbf{R}^{N}} e^{-|y|^{2} /(1-m)} d y\right)^{1-m}+4\left(\int_{\mathbf{R}^{N}}|y|^{2 /(1-m)} e^{-|y|^{2} /(1-m)} d y\right)^{1-m}\right\} .
\end{aligned}
$$

Since $1-(1-m) N / 2>0$ by assumption (4), the right-hand side goes to zero as $\epsilon \downarrow 0$. Thus, the Lebesgue dominated convergence theorem shows equation (10).

Next, let $a(\tau)$ be as above. As is easily seen, the initial-value problem

$$
\begin{cases}\partial_{t} w=\Delta w^{m}+a(\tau) w, & (x, t) \in \mathbf{R}^{N} \times(0, \infty) \\ w(x, 0)=u_{0}(x), & x \in \mathbf{R}^{N}\end{cases}
$$

has a global solution $w$ which also satisfies

$$
\int_{\mathbf{R}^{N}} w(x, t) d x=e^{a(\tau) t} \int_{\mathbf{R}^{N}} u_{0}(x) d x .
$$

Since $w$ is a supersolution to (1)-(2), we conclude (11).

We define $J(t), t \geq 0$, as

$$
J(t)=\left(\int_{\mathbf{R}^{N}} e^{-\epsilon|x|^{2}} d x\right)^{-1} \int_{\mathbf{R}^{N}} u(x, t) e^{-\epsilon|x|^{2}} d x .
$$

Proposition 2.3. If $u_{0}$ is large enough to satisfy

$$
J(0)>(2 N \epsilon)^{1 /(p-m)},
$$

then the corresponding solution $u$ of (1)-(2) is not global. More precisely, there exists $a T>0$ such that

$$
\sup _{x \in \mathbf{R}^{N}} u(x, t) \rightarrow \infty \quad \text { as } \quad t \uparrow T .
$$


Proof. The assertion is already proved in [10, Proposition 2.3]. Namely, it follows from (9) that

$$
J(\tau)-J(0) \geq\left(\int_{\mathbf{R}^{N}} e^{-\epsilon|x|^{2}} d x\right)^{-1} \int_{0}^{\tau} \int_{\mathbf{R}^{N}}\left\{-2 N \epsilon u^{m}+u^{p}\right\} e^{-\epsilon|x|^{2}} d x d t .
$$

Put $\Gamma(\xi)=-2 N \epsilon \xi^{m}+\xi^{p}$. Then it is convex in $\xi \geq 0$, and is positive and increasing in $\xi$ if $\xi>(2 N \epsilon)^{1 /(p-m)}$. So, by the Jensen inequality,

$$
J(\tau) \geq J(0)+\int_{0}^{\tau} \Gamma(J(t)) d t
$$

from which we have

$$
t \leq \int_{J(0)}^{J(t)} \frac{d \xi}{\Gamma(\xi)} \leq \int_{J(0)}^{\infty} \frac{d \xi}{-2 N \epsilon \xi^{m}+\xi^{p}}<\infty
$$

as long as $u(x, t)$ exists. This leads to a contradiction if the solution is global.

Moreover, taking account of (11) of Proposition 2.2, we conclude (13).

We shall close this section by giving a concrete expression of the Barenblatt solution to the initial-value problem

$$
\begin{cases}\partial_{t} v=\Delta v^{m}, & (x, t) \in \mathbf{R}^{N} \times(0, \infty), \\ v(x, 0)=L \delta(x), & x \in \mathbf{R}^{N}\end{cases}
$$

where $L>0$ and $\delta(x)$ is Dirac's $\delta$-function.

Let

$$
\ell=\left(m-1+\frac{2}{N}\right)^{-1}=\left(p_{m}^{*}-1\right)^{-1}>0
$$

and

$$
G_{m}(s)=\left[A+B s^{2}\right]^{-1 /(1-m)}
$$

where $B=\frac{(1-m) \ell}{2 m N}>0$ and $A>0$ is chosen to satisfy $\int_{\mathbf{R}^{N}} G_{m}(|x|) d x=1$.

Proposition 2.4. The solution to (14) is given by

$$
E_{m}(x, t ; L)=L\left(L^{m-1} t\right)^{-\ell} G_{m}\left(|x|\left(L^{m-1} t\right)^{-\ell / N}\right)
$$

and it is self-similar in the following sense: for any $k>0$

$$
k^{N} E_{m}\left(k x, k^{N / \ell} t ; L\right)=E_{m}(x, t ; L)
$$

Proof. The expression (16) is well-known, and the self-similarity (17) easily follows from (15) and (16). 


\section{Proof of Theorem 1.1}

In this section, Theorem 1.1 will be proved in a series of lemmas.

Lemma 3.1. Let $u$ be a global solution of (1)-(2). Then we have

$$
\int_{\mathbf{R}^{N}} u(x, t) e^{-\epsilon|x|^{2}} d x \leq C(N) \epsilon^{-N / 2+1 /(p-m)},
$$

for any $t \geq 0$ and $\epsilon>0$, where $C(N)=\pi^{N / 2}(2 N)^{1 /(p-m)}$.

Proof. Since

$$
\int_{\mathbf{R}^{N}} e^{-\epsilon|x|^{2}} d x=\epsilon^{-N / 2} \int_{\mathbf{R}^{N}} e^{-|y|^{2}} d y=\pi^{N / 2} \epsilon^{-N / 2},
$$

the blow-up condition (12) is reduced to

$$
\int_{\mathbf{R}^{N}} u_{0}(x) e^{-\epsilon|x|^{2}} d x>\pi^{N / 2} \epsilon^{-N / 2}(2 N \epsilon)^{1 /(p-m)}=C(N) \epsilon^{-N / 2+1 /(p-m)} .
$$

Thus, every global solution $u$ must satisfy the converse inequality (18).

Lemma 3.2. Assume that $u$ is global. If $1<p<p_{m}^{*}$, then

$$
\int_{\mathbf{R}^{N}} u(x, t) d x=0 \text { for any } t \geq 0 .
$$

If $p=p_{m}^{*}$, then

$$
\int_{\mathbf{R}^{N}} u(x, t) d x \leq C(N) \text { for any } t \geq 0 .
$$

Proof. Since $u(\cdot, t) \in L^{1}\left(\mathbf{R}^{N}\right)$, noting (3) and letting $\epsilon \downarrow 0$ in (18), we easily have the assertions of the lemma.

Lemma 3.3. Let $p=p_{m}^{*}$. Assume that $u$ is global. Then we have for any $t>0$,

$$
\int_{0}^{t} \int_{\mathbf{R}^{N}} u(x, \tau)^{p} d x d \tau \leq C(N)
$$

Proof. The assertion directly follows from (10) of Proposition 2.2 and (20).

The following lemma is due to Friedman-Kamin [4, Remark 2].

Lemma 3.4. Let $u_{0}(x) \in L^{1}\left(\mathbf{R}^{N}\right) \cap L^{\infty}\left(\mathbf{R}^{N}\right)$, and let $v$ be the solution of the Cauchy problem

$$
\begin{cases}\partial_{t} v=\Delta v^{m}, & (x, t) \in \mathbf{R}^{N} \times(0, \infty), \\ v(x, 0)=u_{0}(x), & x \in \mathbf{R}^{N}\end{cases}
$$

If we put $v_{k}(x, t)=k^{N} v\left(k x, k^{N / \ell} t\right)$, then

$$
v_{k}(x, t) \rightarrow E_{m}(x, t ; L) \quad \text { as } \quad k \rightarrow \infty
$$

locally uniformly in $\mathbf{R}^{N} \times(0, \infty)$, where $L=\int_{\mathbf{R}^{N}} u_{0}(x) d x$. 
Proof of Theorem 1.1. Contrary to the conclusion, assume that for given $u_{0}(x) \not \equiv 0$, the Cauchy problem (1)-(2) has a nontrivial global solution $u$.

In case $1<p<p_{m}^{*}$, a contradiction occurs by (19) of Lemma 3.2 since it implies $u(x, t) \equiv 0$. So, we have only to consider the critical case $p=p_{m}^{*}$.

We put $u_{k}(x, t)=k^{N} u\left(k x, k^{N / \ell} t\right)$. Then it satisfies (1)-(2) with $u_{0}(x)$ replaced by $k^{N} u_{0}(k x)$. Similarly, $v_{k}$ given in Lemma 3.4 satisfies $(22)$ with $u_{0}(x)$ replaced by $k^{N} u_{0}(k x) . u_{k}$ being global, we have from Lemma 3.3

$$
\int_{0}^{t} \int_{\mathbf{R}^{N}} u_{k}(x, \tau)^{p} d x d \tau \leq C(N) \text { for } t>0 .
$$

By definition $v_{k}(x, t) \leq u_{k}(x, t)$ in $\mathbf{R}^{N} \times(0, \infty)$. Thus, it follows from Lemma 3.4 and the Fatou lemma that

$$
\int_{\delta}^{t} \int_{\mathbf{R}^{N}} E_{m}(x, \tau ; L)^{p} d x d \tau \leq \liminf _{k \rightarrow \infty} \int_{\delta}^{t} \int_{\mathbf{R}^{N}} v_{k}(x, \tau)^{p} d x d \tau \leq C(N)
$$

for any $0<\delta<t$.

On the other hand, it follows from Proposition 2.4 that

$$
\int_{\delta}^{t} \int_{\mathbf{R}^{N}} E_{m}(x, \tau ; L)^{p} d x d \tau=L^{p} \int_{\delta}^{t}\left(L^{m-1} \tau\right)^{-p \ell}\left(L^{m-1} \tau\right)^{\ell} d \tau \int_{\mathbf{R}^{N}} G_{m}(|x|)^{p} d x .
$$

Since $p=p_{m}^{*}$, we have $-\ell(p-1)=-1$ in this equality. So, the right side goes to $\infty$ if we let $\delta \rightarrow 0$ or $t \rightarrow \infty$.

This contradicts (24), and the proof of Theorem 1.1 is completed.

\section{Proof of Theorem $\mathbf{1 . 2}$}

In this section, we assume $p>p_{m}^{*}$ in (1). We shall show Theorem 1.2 by constructing a supersolution of (1) in the form

$$
Z(x, t)=\left(t+t_{0}\right)^{-\alpha}\left[a+b|x|^{2}\left(t+t_{0}\right)^{-2 \nu \alpha}\right]^{-1 /(1-m)},
$$

where $t_{0}, a, b, \alpha$, and $\nu$ are suitably chosen positive constants.

Substitute this in the inequality

$$
\partial_{t} Z-\Delta Z^{m} \geq Z^{p}
$$

Then putting

$$
X=a+b|x|^{2}\left(t+t_{0}\right)^{-2 \nu \alpha},
$$

we have

$$
\begin{gathered}
X^{-1 /(1-m)-1}\left\{-\alpha a\left(t+t_{0}\right)^{-\alpha-1}+\frac{2 m N a b}{1-m}\left(t+t_{0}\right)^{-(m+2 \nu) \alpha}\right. \\
\quad-\alpha\left(1-\frac{2 \nu}{1-m}\right) b|x|^{2}\left(t+t_{0}\right)^{-(1+2 \nu) \alpha-1} \\
\left.\quad+\frac{2 m b^{2}}{1-m}\left(N-\frac{2}{1-m}\right)|x|^{2}\left(t+t_{0}\right)^{-(m+4 \nu) \alpha}\right\} \\
\geq X^{-1 /(1-m)-1}\left(t+t_{0}\right)^{-\alpha p}\left[a+b|x|^{2}\left(t+t_{0}\right)^{-2 \nu \alpha}\right]^{-(p+m-2) /(1-m)} .
\end{gathered}
$$


We put $\alpha+1=(m+2 \nu) \alpha=\alpha p$, i.e., $\alpha=\frac{1}{p-1}$ and $\nu=\frac{p-m}{2}$. Then

$$
\begin{aligned}
\text { left side }= & X^{-1 /(1-m)-1}\left(t+t_{0}\right)^{-\alpha p}\{\ldots\} \\
\{\cdots\}= & -\frac{a}{p-1}+\frac{2 m N a b}{1-m}+\frac{1}{1-m} X \\
& +\frac{2 m b}{1-m}\left(N-\frac{2}{1-m}\right) X-\frac{a}{1-m}-\frac{2 m a b}{1-m}\left(N-\frac{2}{1-m}\right) .
\end{aligned}
$$

Thus, it follows from (26) that

$$
\begin{gathered}
F(X) \equiv X^{-(p+m-2) /(1-m)}-\frac{1}{1-m}\left\{1+2 m b\left(N-\frac{2}{1-m}\right)\right\} X \\
+\frac{a}{1-m}\left\{\frac{p-m}{p-1}-\frac{4 m b}{1-m}\right\} \leq 0 .
\end{gathered}
$$

This inequality holds if $a$ and $b$ are chosen to satisfy $F(a) \leq 0$ and $F^{\prime}(X) \leq 0$ in $X \geq a$. The first condition is reduced to

$$
0<a^{-(p-1) /(1-m)} \leq \frac{1}{1-m}\left\{2 m N b-\frac{1-m}{p-1}\right\}
$$

and the second condition is

$$
F^{\prime}(X)=-\frac{p+m-2}{1-m} X^{-(p-1) /(1-m)}-\frac{1}{1-m}\left\{1-\frac{2 m b N\left(p_{m}^{*}-1\right)}{1-m}\right\} \leq 0
$$

in $X \geq a$. If $p+m-2 \geq 0$, then (28) is reduced to

$$
b \leq \frac{1-m}{2 m N\left(p_{m}^{*}-1\right)},
$$

and if $p+m-2<0$, then (28) is reduced to

$$
0<a^{-(p-1) /(1-m)} \leq \frac{-(1-m)+2 m b N\left(p_{m}^{*}-1\right)}{(1-m)(p+m-2)} .
$$

Summarizing these results, we obtain

Lemma 4.1. (i) In case $p+m-2 \geq 0$, let

$$
\frac{1-m}{2 m N(p-1)}<b \leq \frac{1-m}{2 m N\left(p_{m}^{*}-1\right)} .
$$

Then (27) and (29) hold if a is chosen sufficiently large.

(ii) In case $p+m-2<0$, let

$$
\frac{1-m}{2 m N(p-1)}<b<\frac{1-m}{2 m N\left(p_{m}^{*}-1\right)} .
$$

Then (27) and (30) hold if a is chosen sufficiently large. 
Remark. The case (ii) occurs if

$$
\max \left\{0,1-\frac{2}{N}\right\}<m<1-\frac{1}{N} \quad \text { and } \quad p_{m}^{*}<p<2-m,
$$

and the case (i) occurs for other pairs $\{m, p\}$.

Proof of Theorem 1.2. Let $t_{0}>0$, and let $\{a, b\}$ be a pair of positive numbers satisfying the conditions of Lemma 4.1. Then the function

$$
Z(x, t)=\left(t+t_{0}\right)^{-1 /(p-1)}\left[a+b|x|^{2}\left(t+t_{0}\right)^{-(p-m) /(p-1)}\right]^{-1 /(1-m)}
$$

satisfies (25), that is, it becomes a supersolution of (1). Let

$$
u_{0}(x) \leq C\left(1+|x|^{2}\right)^{-1 /(1-m)}
$$

If we choose $t_{0}>0$ and $C>0$ very small to satisfy

$$
0<t_{0} \leq\left(a^{-1} b\right)^{(p-1) /(p-m)} \text { and } C \leq\left(b^{-1} t_{0}\right)^{1 /(1-m)},
$$

then we have

$$
0 \leq u_{0}(x) \leq Z(x, 0), \quad x \in \mathbf{R}^{N} .
$$

Let $u$ be the solution of (1)-(2) with this initial datum $u_{0}(x)$. Then by the comparison principle, it follows that

$$
u(x, t) \leq Z(x, t)
$$

as long as $u(x, t)$ exists. This and (11) of Proposition 2.2 ensure simultaneously the global existence and the decay property (7) of Theorem 1.2.

\section{Exterior Dirichlet problem}

In this section, we shall remark that the critical blow-up occurs also for the exterior Dirichlet problem if the space dimension $N \geq 3$.

Let $E_{R}=\left\{x \in \mathbf{R}^{N} ;|x|>R\right\}(R>0)$, and let us consider the exterior initialboundary-value problem

$$
\begin{cases}\partial_{t} u=\Delta u^{m}+u^{p}, & (x, t) \in E_{R} \times(0, T) \\ u(x, 0)=u_{0}(x), & x \in E_{R} \\ u(x, t)=0, & (x, t) \in \partial E_{R} \times(0, T),\end{cases}
$$

where $m$ satisfies (4), $p=p_{m}^{*}$ and

$$
u_{0}(x) \in L^{1}\left(E_{R}\right) \cap L^{\infty}\left(E_{R}\right) .
$$

In this case, Lemma 3.4 is extended as follows:

Lemma 5.1. Let $v$ be the solution of

$$
\begin{cases}\partial_{t} v=\Delta v^{m}, & (x, t) \in E_{R} \times(0, T), \\ v(x, 0)=u_{0}(x), & x \in E_{R} \\ v(x, t)=0, & (x, t) \in \partial E_{R} \times(0, T) .\end{cases}
$$

If we put $v_{k}(x, t)=k^{N} v\left(k x, k^{N / \ell} t\right), k>0$, then

$$
v_{k}(x, t) \rightarrow E_{m}(x, t ; L) \quad \text { as } \quad k \rightarrow \infty
$$


locally uniformly in $\left\{\mathbf{R}^{N} \backslash\{0\}\right\} \times(0, \infty)$, where

$$
L=\int_{E_{R}} u_{0}(x) \sigma_{R}(|x|) d x, \quad \sigma_{R}(r)=\frac{r^{N-2}-R^{N-2}}{r^{N-2}} .
$$

Proof. As in the whole space case (see [4]), we see that for any $\delta>0$ there exists $k_{\delta}>0$ such that $\left\{v_{k}(x, t) ; k \geq k_{\delta}\right\}$ is uniformly bounded and equicontinuous in $E_{\delta} \times[\delta, \infty)$. Thus, using the Ascoli-Arzela theorem and a diagonal sequence method in $\delta$, we see that for any sequence $\left\{k_{j}\right\} \uparrow \infty$, there exists a subsequence $\left\{k_{j}^{\prime}\right\}$ and a function $w(x, t) \in C\left(\left\{\mathbf{R}^{N} \backslash\{0\}\right\} \times(0, \infty)\right)$ such that

$$
v_{k_{j}^{\prime}}(x, t) \rightarrow w(x, t) \quad \text { as } \quad k_{j}^{\prime} \rightarrow \infty
$$

locally uniformly in $\left\{\mathbf{R}^{N} \backslash\{0\}\right\} \times(0, \infty)$.

To show $w(x, t)=E_{m}(x, t, ; L)$, we can follow the argument of Suzuki $[13, \S 3]$.

$v_{k}$ satisfies equations $(32)$ with $E_{R}$ and $u_{0}(x)$ replaced by $E_{R / k}$ and $k^{N} u_{0}(k x)$, respectively. Thus,

$$
\begin{aligned}
& \int_{E_{R / k}} v_{k}(x, \tau) \varphi(x, \tau) d x-\int_{E_{R / k}} k^{N} u_{0}(k x) \varphi(x, 0) d x \\
&=\int_{0}^{\tau} \int_{E_{R / k}}\left\{v_{k} \partial_{t} \varphi+v_{k}^{m} \Delta \varphi\right\} d x d t
\end{aligned}
$$

for any $\varphi(x, t) \in C_{0}^{2}\left(\bar{E}_{R / k} \times[0, \infty)\right)$ such that $\varphi(x, t)=0$ on $|x|=R / k$. We choose $\varphi(x, t)=\sigma_{R / k}(|x|) \zeta(x, t)$, where $\zeta(x, t) \in C_{0}^{2}\left(\mathbf{R}^{N} \times[0, \infty)\right)$, in this equation, and let $k=k_{j}^{\prime} \rightarrow \infty$. It then follows that

$$
\int_{\mathbf{R}^{N}} w(x, \tau) \zeta(x, \tau) d x-\zeta(0,0) L=\int_{0}^{\tau} \int_{\mathbf{R}^{N}}\left\{w \partial_{t} \zeta+w^{m} \Delta \zeta\right\} d x d t
$$

for $\tau>0$, where $L$ is as given in (34).

Note that (35) is satisfied by the Barenblatt solution $E_{m}(x, t ; L)$. Then the uniqueness theorem due to Dahlberg-Kenig [3] (cf. also Pierre [12]) implies the desired equality.

Now, let $u$ be a global solution to (31). Then since $u_{k}=k^{N} u\left(k x, k^{N / \ell} t\right)$ is again a global solution to

$$
\begin{cases}\partial_{t} u_{k}=\Delta u_{k}^{m}+u_{k}^{p}, & (x, t) \in E_{R / k} \times(0, T) \\ u_{k}(x, 0)=k^{N} u_{0}(k x), & x \in E_{R / k} \\ u_{k}(x, t)=0, & (x, t) \in \partial E_{R / k} \times(0, T)\end{cases}
$$

it follows from our blow-up conditions that

$$
\int_{\delta}^{\tau} \int_{E_{R / k}} u_{k}(x, t)^{p} \rho_{R / k}(|x|) d x d t \leq(2 N+4)^{1 /(p-m)} \pi^{N / 2}
$$

for any $0<\delta<t<\infty$, where $\rho_{R / k}(r)=\frac{r-(R / k)}{r}$ (cf., [10, §4]). The number $(2 N+4)$ corresponds to $2 N$ in (12) or (18), and it appears when we use the inequality

$$
\Delta\left[\rho_{R / k}(|x|) e^{-\epsilon(|x|-R / k)^{2}}\right] \geq-(2 N+4) \epsilon \rho_{R / k}(|x|) e^{-\epsilon(|x|-R / k)^{2}} .
$$


Suppose that $u$ is nontrivial. Then combining (36) and Lemma 5.1, we can follow the proof of Theorem 1.1 to yield a contradiction.

The critical blow-up for the exterior problem (31) is thus concluded.

\section{References}

1. J. G. Berryman, Evolution of a stable profile for a class of nonlinear diffusion equations with fixed boundary, J. Math. Phys. 18 (1977), 2108-2115.

2. H. Brézis and M. G. Crandall, Uniqueness of solutions of the initial-value problem for $u_{t}-$ $\Delta \varphi(u)=0$, J. Math. Pure et Appl. 58 (1979), 153-163.

3. B. E. J. Dahlberg and C. E. Kenig, Non-negative solutions to fast diffusions, Revista Math. Iberoamericana 4 (1988), 11-29.

4. A. Friedman and S. Kamin, The asymptotic behavior of gas in an $n$-dimensional porous medium, Trans. Amer. Math. Soc. 262 (1980), 551-563.

5. H. Fujita, On the blowing up of solutions of the Cauchy problem for $u_{t}=\Delta u+u^{1+\sigma}$, J. Fac. Sci. Univ. Tokyo 13 (1966), 109-124.

6. V. A. Galaktionov, Blow-up for quasilinear heat equations with critical Fujita's exponents, to appear Proc. Roy. Soc. Edinburgh.

7. V. A. Galaktionov, S. P. Kurdyumov, A. P. Mikhailov, and A. A. Samarskii, Unbounded solutions of the Cauchy problem for the parabolic equation $u_{t}=\nabla\left(u^{\sigma} \nabla u\right)+u^{\beta}$, Soviet Phys. Dokl. 25 (1980), 458-459.

8. K. Hayakawa, Non existence of global solutions of some semilinear parabolic equations, Proc. Japan Acad. 49 (1973), 503-505.

9. T. Kawanago, Existence and behavior of solutions for $u_{t}=\Delta\left(u^{m}\right)+u^{\ell}$, Preprint.

10. K. Mochizuki and R. Suzuki, Critical exponent and critical blow-up for quasilinear parabolic equations, Preprint.

11. L. A. Peletier, The porous media equation, Applications of Nonlinear Analysis in the Physical Sciences, Pitman, London, 1981, 229-241.

12. M. Pierre, Uniqueness of the solutions of $u_{t}-\Delta \varphi(u)=0$ with initial datum a measure, Nonlinear Anal. T. M. A. 6 (1982), 175-187.

13. R. Suzuki, Critical blow-up for quasilinear parabolic equations in exterior domains, Preprint.

14. F. B. Weissler, Existence and nonexistence of global solutions for a semilinear heat equation, Israel J. Math. 38 (1981), 29-40.

Department of Mathematics, Tokyo Metropolitan University, Hachioji, Tokyo 192-03, JAPAN 